\title{
Germanica
}

$47 \mid 2010$

« Krack! Tschock ! Pflatsch ! Bummmm ! La BD de langue allemande (à suivre...) »

\section{Marion Linhardt (Hrsg.), Stimmen zur Unterhaltung. Operette und Revue in der publizistischen Debatte (1906-1933)}

\section{Marc Lacheny}

\section{(2) OpenEdition}

\section{Journals}

Édition électronique

URL : http://journals.openedition.org/germanica/1152

DOI : 10.4000/germanica. 1152

ISSN : 2107-0784

Éditeur

Université de Lille

Édition imprimée

Date de publication : 31 décembre 2010

Pagination : 193-196

ISBN : 9782913857261

ISSN : 0984-2632

Référence électronique

Marc Lacheny, "Marion Linhardt (Hrsg.), Stimmen zur Unterhaltung. Operette und Revue in der

publizistischen Debatte (1906-1933) », Germanica [En ligne], 47 | 2010, mis en ligne le 08 juillet 2011 consulté le 06 octobre 2020. URL : http://journals.openedition.org/germanica/1152 ; DOI : https:// doi.org/10.4000/germanica.1152

Ce document a été généré automatiquement le 6 octobre 2020 .

(c) Tous droits réservés 


\title{
Marion Linhardt (Hrsg.), Stimmen zur Unterhaltung. Operette und Revue in der publizistischen Debatte (1906-1933)
}

\author{
Marc Lacheny
}

1 Marion Linhardt présente ici une anthologie de 51 articles qui documentent en profondeur l'évolution du «théâtre de divertissement musical» (musikalisches Unterhaltungstheater, p. 9) en Europe centrale de 1905-1906 à 1933 (date de la prise du pouvoir par les nationaux-socialistes en Allemagne).

2 L'ouvrage débute par une introduction particulièrement utile et substantielle, sobrement intitulée "Einblicke in den Theateralltag der Moderne", de l'éditrice (p. 11-37). Si Marion Linhardt y précise dans un premier temps les notions d'« avantgarde » et de "modernité » (p. 11), c'est pour mieux poser, puis éclairer les enjeux du vaste débat sur l'opérette et la revue à grand spectacle qui a lieu au cours des premières décennies $\mathrm{du} \mathrm{xx}^{\mathrm{e}}$ siècle. Elle reconnaît à juste titre dans le vif succès rencontré fin décembre 1905 par La Veuve joyeuse de Franz Lehár et Victor Léon un tournant décisif dans l'évolution du genre de l'opérette : avec cette œuvre, l'opérette perd sa coloration locale viennoise, issue de l'opéra comique, pour prendre une dimension internationale et transatlantique constitutive du «théâtre de divertissement de la modernité » (p. 14). Aussi la suite de l'introduction est-elle fort logiquement consacrée à ce processus d'« internationalisation » de l'opérette qui s'amorce dès les années 1890 , pour devenir en quelque sorte "le genre de la Mitteleuropa» (p. 23). Marion Linhardt souligne par ailleurs le rôle d'un " acteur " émergent majeur dans ce contexte, celui du metteur en scène (Regisseur), appelé à utiliser le libretto et la musique de l'opérette comme un matériel lui permettant de développer sa propre démarche artistique (p.29). L'introduction s'achève par une mise en regard convaincante des phénomènes de concurrence et d'interactions (surtout à partir des années 1920) entre l'opérette et la revue à grand spectacle à Berlin, Londres, Paris et Vienne: une importance toute 
particulière est ici accordée à la revue à Berlin dans les années 1920, ainsi qu'à l'adoption par l'opérette de techniques propres à la revue (p. 35-37).

3 En dépit de la variété des points de vue, des méthodes et des styles, parfois antithétiques qui s'y expriment, la plupart des articles retenus dans cette anthologie tentent de répondre à la question de savoir si l'opérette doit se muer en une forme esthétique profonde, placée sous le signe de la psychologie et de l'exigence, ou si elle doit au contraire, conformément à sa nature première, privilégier le divertissement du plus grand nombre aux dépens de toute prétention artistique.

L'anthologie de Marion Linhardt débute (p.39-45) par un article de l'écrivain, journaliste et critique Felix Salten (1869-1945) intitulé « Die neue Operette » et paru dans la revue viennoise Die Zeit en décembre 1906, soit près d'un an après la première de La Veuve joyeuse au Theater an der Wien. Dans ce "texte programmatique de l'opérette moderne » (p. 15), Salten apparaît clairement comme un thuriféraire de la "nouvelle opérette", symbole à ses yeux d'une synthèse réussie entre approfondissement psychologique et divertissement d'un large public.

Dans son article «Operetten-Betrachtungen» de 1910 (p. 60-65), le compositeur Oscar Straus (1870-1954) met, quant à lui, à profit la première triomphale de son opérette Ein Walzertraum à Paris en 1910 pour comparer la position du compositeur d'opérettes à Vienne et à Paris. Le texte de Karl Kraus "Ernst ist das Leben, heiter die Operette " (p.65-68) permet au lecteur de poursuivre cette réflexion, le polémiste prenant clairement parti pour l'opérette satirique parisienne d'offenbach aux dépens de la nouvelle opérette viennoise - jugée sirupeuse, décadente et commerciale - incarnée par Lehár. Dans son article « Operette und Revue. Diagnose ihres Zustandes » de 1929 (p. 268-276), le compositeur Ernst Krenek (1900-1991) accrédite la thèse krausienne d'un déclin du genre de l'opérette (viennoise), qu'il impute pour sa part à un recours abusif au kitsch. Cette thèse d'un déclin de l'opérette est d'ailleurs commune aux textes de Willy Werner Göttig ("Der Verfall der Operette », 1925, p. 152-164), de Karl Neisser («Die sterbende Operette », 1927, p. 235-237), de Max Steiner-Kaiser (« Zum Niedergang der Operette ", 1929, p. 291-296), de Georg Pauly et de Harry Kahn ("Operettendämmerung», 1912 et 1928, p. 77-85 et 247-251). Au chapitre des voix critiques qui s'élèvent au sujet de la «nouvelle » opérette, il convient d'ajouter les articles de Hans Joachim Moser et de Josef Stolzing qui, dans leurs titres mêmes («Die Operettenepidemie », 1914, p. 107-115, et «Die Operettenseuche», 1917, p. 125-131), empruntent largement au registre de la maladie pour marquer leur défiance à l'égard de la «nouvelle » opérette. Au texte de Krenek évoqué plus haut succède (p. 276-283) un article fort instructif de Herbert Graf (1903-1973), metteur en scène célèbre, intitulé «Bearbeitung und Neuinszenierung alter Operetten» (1929). Graf y rejoint les propos polémiques de Kraus et de Krenek sur l'opérette moderne, dans laquelle il considère deux des caractéristiques initiales du genre, l'humour et le divertissement, comme détruites - ce qui le pousse à réclamer l'adaptation et l'actualisation d'opérettes anciennes.

Un autre motif fondamental de désaccord au sein du débat publiciste tient au phénomène d'érotisation massive de l'opérette au tournant $\mathrm{du} \mathrm{xx}^{\mathrm{e}}$ siècle : tandis que Salten voyait précisément dans cette "Enthüllung des Triebhaften» (p.42) un des signes de la modernisation du genre, Georg Pauly (1883-1950) y reconnaît au contraire la marque d'un déclin moral, « ein lügenhaftes Zerrbild [...], wenn sie immer wieder den Männern die zweifelhafte Eleganz grossstädtischer Nachtlokale und den Frauen 
Männerfang mit Dirnenmitteln als das erstrebenswerteste Ziel des Lebens hinstellt » (p.83). On peut tracer ici une ligne de démarcation nette entre les opérettes «nouvelles », sociales, de Lehár, Oscar Straus, Leo Fall, Emmerich Kálmán, Heinrich Reinhardt ou Robert Winterberg d'une part - mettant en œuvre, comme le souligne Salten dès 1906 (p. 44), une véritable "dramaturgie du corps" (sexualité, danse, pantomime) - et l'«opérette légère", comique, de Friedrich von Flotow, Albert Lortzing ou Otto Nicolai d'autre part - héritée de l'opérette viennoise de la fin du XIX siècle (Strauss, Suppè, Millöcker...) et ancrée dans la tradition de l'opéra comique et du théâtre populaire viennois. Avec Fall, Lehár ou Straus, l'opérette viennoise passe ainsi du domaine comique au domaine érotique. C'est également dans ce contexte d'érotisation de l'opérette que s'inscrivent les déclarations souvent radicales émises dans la presse viennoise de l'époque contre la «laideur » des « danses nègres » de Joséphine Baker à Vienne en 1928. D'une telle position à la «mise au pas» (Gleichschaltung)de la création artistique par les Nazis (fustigation, puis destruction de l'art moderne, jugé " dégénéré » (entartet), et culte d'un art nazi faisant l'apologie du sang, de la race et de la terre (arteigene Kunst)), il n'y avait qu'un pas...

7 Marion Linhardt a opté pour un vaste échantillon de voix et de formes d'expression (musicales, littéraires, journalistiques). On aurait pu craindre de prime abord que cette polyphonie accouchât sinon d'une cacophonie, du moins d'une relative dysharmonie... Il n'en est rien. Au contraire, c'est précisément la diversité des voix et des approches qui fait la richesse d'une anthologie dont la cohérence se voit encore renforcée par le soin apporté par l'éditrice à la présentation de son ouvrage : on y trouve d'abord une introduction, exemplaire et d'une lecture aisée, qui fait immédiatement pénétrer le lecteur au cœur du propos, puis des annotations, à la fois succinctes et précises, sur chaque nouvel auteur introduit dans le recueil, et enfin un registre exhaustif des noms et des œuvres cités (p. 327-336). Quelques noms ont été écorchés : ainsi Le Postillon de Longjumeau d'Adolphe Adam (1836) apparait-il ici sous la forme Le Postillon de Lonjumeau et le prénom de Bizet, Georges, sous la forme féminine de George (p. 327).

8 Ce ne sont là, néanmoins, que de menus détails qui n'ôtent rien aux qualités de cet ouvrage qu'aucun spécialiste un tant soit peu sérieux de l'opérette et du «théâtre de divertissement" dans la Vienne de la première moitié $d u x^{e}$ siècle ne pourra désormais se permettre d'ignorer. Il vient, en outre, compléter l'admirable travail d'habilitation de Marion Linhardt, publié sous le titre Residenzstadt und Metropole. $\mathrm{Zu}$ einer kulturellen Topographie des Wiener Unterhaltungstheaters (1858-1918) (Tübingen, Niemeyer, 2006), ainsi que "Warum es der Operette so schlecht geht 》- Ideologische Debatten um das musikalische Unterhaltungstheater (1880-1916), édité là encore par Marion Linhardt (Wien, Köln, Weimar, 2001 = Maske und Kothurn 45, Heft 1-2). 